\title{
O035: Third federal state wide survey on MRSA management in North Rhine-Westphalian hospitals
}

\author{
A Jurke ${ }^{1 *}$, R Köck ${ }^{2}$ AW Friedrich ${ }^{3}$, R Kämmerer ${ }^{4}$, I Daniels-Haardt ${ }^{5}$ \\ From 2nd International Conference on Prevention and Infection Control (ICPIC 2013) \\ Geneva, Switzerland. 25-28 June 2013
}

\section{Introduction}

Staphylococcus aureus (S. aureus) is a major cause of healthcare-associated infections. In Germany in 2011, about $18.2 \%$ of $S$. aureus from blood cultures were methicillin-resistant (MRSA).

\section{Objectives}

In 2011, the Ministry of Health, Emancipation, Care and Age of North Rhine-Westphalia (NRW), initiated the third federal state wide survey in hospitals to inspect the MRSA-management and implementation of the recommendations of the German national Commission for Hospital Hygiene and Infection Control (KRINKO).

\section{Methods}

All hospitals were requested to submit the number of MRSA cases per 1,000 patient-days, the number of colonisations or infections, stratified in imported or nosocomial, the proportion of MRSA isolates in all S. aureus isolates from blood cultures and the number of blood culture samples taken in the year 2011. In addition, the implementation of the KRINKO recommendations in the hospitals has been assessed by local health authorities.

\section{Results}

The response rate was $97.8 \%$; $92.7 \%$ of the 315 hospitals provided analyzable data.

The mean MRSA incidence density was 2.59 per 1,000 patient days; the median was 1.35 with quartiles of 0.89 and 1.99. In 254 hospitals a mean of $21.0 \%$ (median $18.0 \%$, quartiles of 5.9 and 27.0) of all S. aureus detected in blood cultures were MRSA. The hospitals screened in average $21.1 \%$ (median 12.0, lower and upper quartiles of 4.8 and 29.7.) of all patients on admission for the carriage of MRSA. The local health authorities appraised, that $85 \%$ of the responding hospitals have been adequately addressed the national health recommendations.

\section{Conclusion}

The data give insight in MRSA prevalence and management of hospitals providing service to 18 million inhabitants. The study has led to a greater awareness about MRSA in regional hospitals and revealed the progress achieved since 2006. Since 2006 the mean screening rate has nearly quadrupled and the mean MRSA incidence density increased by $36 \%$. The implementation of the KRINKO recommendations must be improved further as well as the data quality. The survey is a pragmatic instrument to monitor the MRSA prevention and control measures in hospitals in a federal state.

\section{Disclosure of interest}

None declared.

\section{Author details}

${ }^{1}$ Infectiology and Hygiene, NRW Centre of Health, Germany. ${ }^{2}$ Institute of Hygiene, University Hospital Münster, Münster, Germany. ${ }^{3}$ Dept. of Medical Microbiology and Infection Prevention, University Medical Center Groningen, Groningen, The Netherlands. ${ }^{4}$ North Rhine Westphalian Ministery of Health, Emancipation, Care and Age, Düsseldorf, Germany. ${ }^{5}$ Health Protection, Health Reporting, NRW Centre of Health, Münster, Germany.

Published: 20 June 2013

doi:10.1186/2047-2994-2-S1-035

Cite this article as: Jurke et al:: 0035: Third federal state wide survey on MRSA management in North Rhine-Westphalian hospitals. Antimicrobial Resistance and Infection Control 2013 2(Suppl 1):035. 